\title{
Polynomial Time Solvable Algorithms to a Class of Unconstrained and Linearly Constrained Binary Quadratic Programming Problems
}

\author{
Shenshen Gu, Rui Cui, and Jiao Peng \\ School of Mechatronic Engineering and Automation \\ Shanghai University \\ 149 Yanchang Road, Shanghai, 200072, P.R.China \\ Email: gushenshen@shu.edu.cn
}

\begin{abstract}
Binary quadratic programming $(B Q P)$ is a typical integer programming problem widely applied in the field of signal processing, economy, management and engineering. However, it is NP-hard and lacks efficient algorithms. Due to this reason, in this paper, some novel polynomial algorithms are proposed to solve a class of unconstrained and linearly constrained binary quadratic programming problems. We first deduce the polynomial time solvable algorithms to the unconstrained binary quadratic programming problems with $Q$ being a seven-diagonal matrix ( $U B Q P 7)$ and a five-diagonal matrix (UBQP5) respectively with two different approaches. Then, the algorithm to unconstrained problem is combined with the dynamic programming method to solve the linearly constrained binary quadratic programming problem with $Q$ being a five-diagonal matrix ( $L C B Q P 5)$. In addition, the polynomial solvable feature of these algorithms are analyzed and some specific examples are presented to illustrate these new algorithms. Lastly, we demonstrate their polynomial feature as well as their high efficiency.
\end{abstract}

(c) 2013 Published by Elsevier Ltd.

Keywords: Binary quadratic programming, Polynomial time solvable algorithm, Dynamic programming

\section{Introduction}

In this paper, we consider the following binary quadratic programming $(B Q P)$ :

$$
\min _{x \in\{0,1\}^{n}} \frac{1}{2} x^{T} Q x+c^{T} x
$$

where $Q=\left(q_{i j}\right)_{n \times n}$ is a symmetric matrix with zero elements in the main diagonal, $c \in \mathbb{R}^{n}$ is a real vector. As the binary quadratic programming problem, $x_{i}$ takes only 0 or 1 . As a result, there is no loss of generality in assuming the zero diagonal because $x_{i}^{2}=x_{i}(1 \leqslant i \leqslant n)$. More specifically, the above problem is also called unconstrained binary quadratic programming $(U B Q P)$. For problem (1) with linear constraint $a^{T} x \leq b\left(a \in \mathbb{Z}_{+}^{n}, b \in \mathbb{Z}_{+}\right)$, it is called linearly constrained binary quadratic programming $(L C B Q P)$. BQP are typical optimization problems which is well known as NP-hard problem (see [1]).

Many applications of $B Q P$ problem exist such as signal processing, financial data analysis [2], molecular conformation [3] and cellular radio channel assignment [4]. Various exact solution methods for solving $B Q P$ and its variants have been proposed in the literature (see, e.g., [4]-[10] and the references therein). Some important methods 
include the decomposition method[4], semidefinite relaxations and cutting planes method to improve the quality of the bounds[8], and semidefinite and polyhedral relaxation method[10].

We focus in this paper on a class of polynomially solvable cases of the quadratic binary programming problems. These cases include the unconstrained binary quadratic programming problems with $Q$ being a five-diagonal matrix and a seven-diagonal matrix (denoted by $U B Q P 5$ and $U B Q P 7$ respectively), and the linearly constrained binary quadratic programming problems with $Q$ being a five-diagonal matrix (denoted by $L C B Q P 5$ ). Identifying polynomially solvable subclasses of problem $B Q P$ not only offers theoretical insight into the complicated nature of the problem, but also provides useful information for designing efficient algorithms for finding optimal solution to problem $B Q P$. More specifically, the properties of the polynomially solvable subclasses provide hints and facilitate the derivation of efficient relaxations for the general form of $B Q P$.

The remainder of this paper is organized as follows: In Section 2, the algorithm to problem $U B Q P 7$ is designed based on the property of matrix $Q$. In Section 3, the algorithm to problem $U B Q P 5$ is designed based on famous basic algorithm $[11,12]$. In Section 4, the algorithm to solve $L C B Q P 5$ is proposed by combining the basic algorithm and the dynamic programming method. And in Section 5, we make an analysis on the polynomial feature of these algorithms. Next, some examples are given in Section 6 to illustrate these algorithms. Then, in order to demonstrate the effectiveness and efficiency of these novel algorithms, the simulation experiments are performed in Section 7. And finally, Section 8 concludes this paper.

\section{Polynomial Solvable Algorithm to UBQP7}

First, in this section, we consider $B Q P 7$ where $Q$ is a seven-diagonal symmetric matrix with zero diagonal elements which takes

$$
\left(\begin{array}{ccccccccc}
0 & q_{12} & q_{13} & q_{14} & \ldots & 0 & 0 & 0 & 0 \\
q_{12} & 0 & q_{23} & q_{24} & \ldots & 0 & 0 & 0 & 0 \\
q_{13} & q_{23} & 0 & q_{34} & \ldots & 0 & 0 & 0 & 0 \\
q_{14} & q_{24} & q_{34} & 0 & \ldots & 0 & 0 & 0 & 0 \\
\vdots & \vdots & \vdots & \vdots & \ddots & \vdots & \vdots & \vdots & \vdots \\
0 & 0 & 0 & 0 & \ldots & 0 & q_{n-3, n-2} & q_{n-3, n-1} & q_{n-3, n} \\
0 & 0 & 0 & 0 & \ldots & q_{n-3, n-2} & 0 & q_{n-2, n-1} & q_{n-2, n} \\
0 & 0 & 0 & 0 & \ldots & q_{n-3, n-1} & q_{n-2, n-1} & 0 & q_{n-1, n} \\
0 & 0 & 0 & 0 & \ldots & q_{n-3, n} & q_{n-2, n} & q_{n-1, n} & 0
\end{array}\right)
$$

For each $x_{i}$, it can be set to either 0 or 1 . That is to say, if we use enumeration method, there will be $2^{n}$ possibilities. With the increasing of the number of dimension, calculation will grow exponentially.

Here, we proposed a novel algorithm to solve $B Q P 7$ in polynomial time based on the property of matrix $Q$. In this algorithm, each time when we set $x_{i}$ to 0 or 1 , at most eight forms of $f(x)$ existed. This can lead to limited calculation, and make the algorithm effective and efficient.

In our proposed algorithm, we first let $i=n$ and set $x_{i}, x_{i-1}$ and $x_{i-2}$ to 0 or 1 , generating eight states of $x$ $\left(x=\left(x_{1}, \ldots, x_{n}\right)\right)$ and the corresponding eight forms of $f(x)$. For every two adjacent states of $x$ (only $x_{i}$ are different, like state (0) and state (1), state (2) and state (3) in Table 1), only two terms in $f(x)$ are different. One is the term contains variable $x_{i-3}$ and the other is the constant term. When we further set $x_{i-3}$ to 0 and 1 , these different two terms can be compared. Thus, we eliminate the bad state and keep the good one for next calculations. We can apply the similar process for the remaining seven states. Therefore, each time when we assign 0 or 1 to $x_{i}$, there will be only eight forms of $f(x)$ left. The flow chart of our algorithm is shown in Fig.1 and the procedures of our algorithm are given in detail as follows:

Procedure 2.1. Generate eight forms of $f(x)$ by assigning 0 or 1 to $x_{i}, x_{i-1}, x_{i-2}$.

Let $i=n$ at the first round of calculation.

Assign (0, 0, 0), (0, 0, 1), (0,1,0), (0,1,1), (1, 0,0), (1,0,1), (1, 1,0), and $(1,1,1)$ to $\left(x_{i-2}, x_{i-1}, x_{i}\right)$ to generate eight forms of $f(x)$, which is summarized in Table 1. 
Table 1. Different states of $f(x)$

\begin{tabular}{|c|c|c|}
\hline states & $\left(x_{i-2}, x_{i-1}, x_{i}\right)$ & $f\left(x_{1}, \ldots, x_{n}\right)$ \\
\hline state (0) & $(0,0,0)$ & $\begin{array}{l}q_{12} x_{1} x_{2}+\ldots+q_{i-4, i-3} x_{i-4} x_{i-3}+q_{i+1, i+2} x_{i+1} x_{i+2}+\ldots+q_{n-1, n} x_{n-1} x_{n} \\
+c_{1} x_{1}+\ldots+c_{i-4} x_{i-4}+c_{i+1} x_{i+1}+\ldots+c_{n} x_{n}+c_{i-3} x_{i-3}\end{array}$ \\
\hline state (1) & $(0,0,1)$ & $\begin{array}{l}q_{12} x_{1} x_{2}+\ldots+q_{i-4, i-3} x_{i-4} x_{i-3}+q_{i+1, i+2} x_{i+1} x_{i+2}+\ldots+q_{n-1, n} x_{n-1} x_{n} \\
+c_{1} x_{1}+\ldots+c_{i-4} x_{i-4}+c_{i+1} x_{i+1}+\ldots+c_{n} x_{n}+\left(c_{i-3}+q_{i-3, i}\right) x_{i-3}+c_{i}\end{array}$ \\
\hline state (2) & $(0,1,0)$ & $\begin{array}{l}q_{12} x_{1} x_{2}+\ldots+q_{i-4, i-3} x_{i-4} x_{i-3}+q_{i+1, i+2} x_{i+1} x_{i+2}+\ldots+q_{n-1, n} x_{n-1} x_{n} \\
+q_{n-1, n} x_{n-1} x_{n}+c_{1} x_{1}+\ldots+c_{i-5} x_{i-5}+c_{i+1} x_{i+1}+\ldots+c_{n} x_{n} \\
+\left(c_{i-4}+q_{i-4, i-1}\right) x_{i-4}+\left(c_{i-3}+q_{i-3, i-1}\right) x_{i-3}+c_{i-1}\end{array}$ \\
\hline state (3) & $(0,1,1)$ & $\begin{array}{l}q_{12} x_{1} x_{2}+\ldots+q_{i-4, i-3} x_{i-4} x_{i-3}+q_{i+1, i+2} x_{i+1} x_{i+2}+\ldots+q_{n-1, n} x_{n-1} n \\
+c_{1} x_{1}+\ldots+c_{i-5} x_{i-5}+c_{i+1} x_{i+1}+\ldots+c_{n} x_{n} \\
+\left(c_{i-4}+q_{i-4, i-1}\right) x_{i-4}+\left(c_{i-3}+q_{i-3, i-1}+q_{i-3, i}\right) x_{i-3}+c_{i-1}+c_{i}\end{array}$ \\
\hline state (4) & $(1,0,0)$ & $\begin{array}{l}q_{12} x_{1} x_{2}+\ldots+q_{i-4, i-3} x_{i-4} x_{i-3}+q_{i+1, i+2} x_{i+1} x_{i+2}+\ldots+q_{n-1, n} x_{n-1} x_{n} \\
+c_{1} x_{1}+\ldots+c_{i-6} x_{i-6}+c_{i+1} x_{i+1}+\ldots+c_{n} x_{n}+\left(c_{i-5}+q_{i-5, i-2}\right) x_{i-5} \\
+\left(c_{i-4}+q_{i-4, i-2}\right) x_{i-4}+\left(c_{i-3}+q_{i-3, i-2}\right) x_{i-3}+c_{i-2}\end{array}$ \\
\hline state (5) & $(1,0,1)$ & $\begin{array}{l}q_{12} x_{1} x_{2}+\ldots+q_{i-4, i-3} x_{i-4} x_{i-3}+q_{i+1, i+2} x_{i+1} x_{i+2}+\ldots+q_{n-1, n} x_{n-1} x_{n} \\
+c_{1} x_{1}+\ldots+c_{i-4} x_{i-4}+c_{i+1} x_{i+1}+\ldots+c_{n} x_{n}+\left(c_{i-5}+q_{i-5, i-2}\right) x_{i-5} \\
+\left(c_{i-4}+q_{i-4, i-2}\right) x_{i-4}+\left(c_{i-3}+q_{i-3, i-2}+q_{i-3, i}\right) x_{i-3}+c_{i-2}+q_{i-2, i}+c_{i}\end{array}$ \\
\hline state (6) & $(1,1,0)$ & $\begin{array}{l}q_{12} x_{1} x_{2}+\ldots+q_{i-4, i-3} x_{i-4} x_{i-3}+q_{i+1, i+2} x_{i+1} x_{i+2}+\ldots+q_{n-1, n} x_{n-1} x_{n} \\
+c_{1} x_{1}+\ldots+c_{i-6} x_{i-6}+c_{i+1} x_{i+1}+\ldots+c_{n} x_{n}+\left(c_{i-5}+q_{i-5, i-2}\right) x_{i-5} \\
+\left(c_{i-4}+q_{i-4, i-2}+q_{i-4, i-1}\right) x_{i-4}+\left(c_{i-3}+q_{i-3, i-2}+q_{i-3, i-1}\right) x_{i-3} \\
+c_{i-2}+q_{i-2, i-1}+c_{i-1}\end{array}$ \\
\hline state (7) & $(1,1,1)$ & $\begin{array}{l}q_{12} x_{1} x_{2}+\ldots+q_{i-4, i-3} x_{i-4} x_{i-3}+q_{i+1, i+2} x_{i+1} x_{i+2}+\ldots+q_{n-1, n} x_{n-1} x_{n} \\
+c_{1} x_{1}+\ldots+c_{i-6} x_{i-6}+c_{i+1} x_{i+1}+\ldots+c_{n} x_{n}+\left(c_{i-5}+q_{i-5, i-2}\right) x_{i-5} \\
+\left(c_{i-4}+q_{i-4, i-2}+q_{i-4, i-1}\right) x_{i-4}+\left(c_{i-3}+q_{i-3, i-2}+q_{i-3, i-1}+q_{i-3, i}\right) x_{i-3} \\
+c_{i-2}+q_{i-2, i-1}+q_{i-2, i}+c_{i-1}+q_{i-1, i}+c_{i}\end{array}$ \\
\hline
\end{tabular}




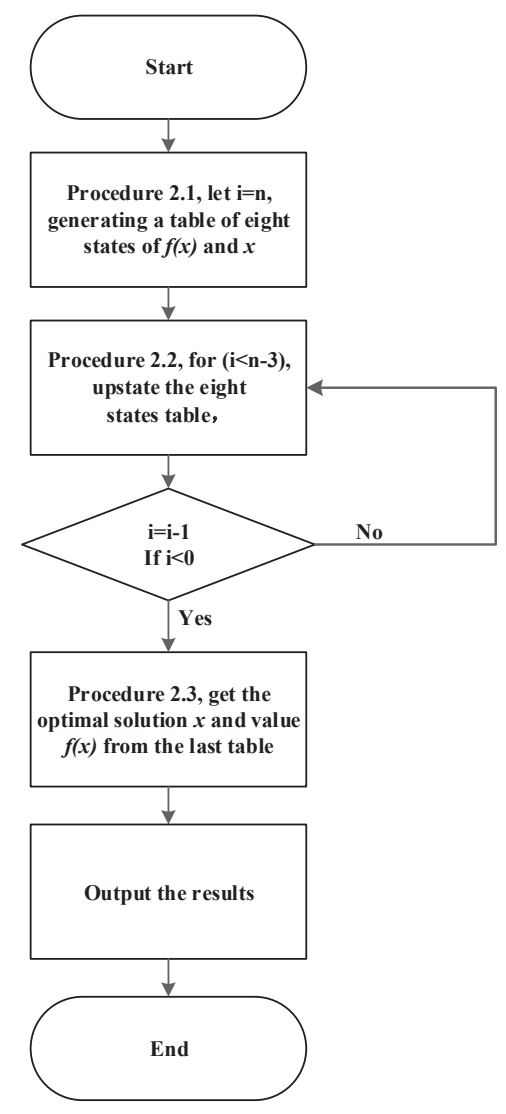

Figure 1. The flow chart of the polynomial solvable algorithm to $U B Q P 7$

Procedure 2.2. Set $x_{i-3}$ to 0 and 1 respectively to generate the new states. This is the key procedure of the whole algorithm.

Step 1: Set $x_{i-3}$ to 0

For state (0) and state (1) in Table 1, they are only different in the linear term coefficient of $x_{i-3}$ and the constant term. Therefore we set $x_{i-3}=0$, calculate $f(x)$ of state $(0)$ and state (1) respectively. Compare these two results to eliminate the bad state and preserve the good one as the new state (0). Similarly, the new state (1) can be generated from state (2) and state (3) by setting $x_{i-3}=0$, the new state (2) is from state (4) and state (5) and the new state (3) is from state (6) and state (7).

Step 2: Set $x_{i-3}$ to 1

By applying the same updating process, we set $x_{i-3}$ to 1 and calculate $f(x)$ of state $(0)$ and state (1). Compare the results and choose the good one as the new state (4). Similarly, we can obtain the new state (5) from state (2) and state (3); the new state (6) from state (4) and state (5); and the new state (7) from state (6) and state (7). By applying the above two steps, we will get a new table of eight forms of $f(x)$ and its corresponding states of $x$.

Procedure 2.3. Set the remaining $x_{i}$ to 0 or 1 .

Set $i$ to $i-1$ and repeat Procedure 2.3, we can generate a table of eight states of $f(x)$ and its corresponding states of $x$ for each time when we set $x_{i}$ to 0 or 1 . After every variable in $x$ is being set to 0 or 1 , the final table contains only constant term of $f(x)$ and its corresponding states of $x$. Choose the optimal value and the corresponding value of $x$ to get the optimal solution. 
Based on the above procedures, we can demonstrate the polynomial solvable algorithm for $U B Q P 7$ as follows:

Algorithm 2.1. Polynomial Solvable Algorithm to UBQP7

Step 1: Let $i=n$ and set $x_{i}, x_{i}-1$, and $x_{i}-2$ to 0 or 1 .

Step 2: $\quad$ Calculate $f(x)$ separately when $\left(x_{i-2}, x_{i-1}, x_{i}\right)$ is assigned to $(0,0,0),(0,0,1),(0,1,0),(0,1,1),(1,0,0)$, $(1,0,1),(1,1,0),(1,1,1)$, generating eight states of $f(x)$.

Step 3: Let $i=n-3$.

Step 4: Set $x_{i}$ to 0 and then compute $f(x)$ of the eight states in Step 2, choose four good states as the new state (0), state (1), state (2) and state (3).

Step 5: Set $x_{i}$ to 1 and then compute $f(x)$ of the eight states in Step 2, choose four good states as the new state (4), state (5), state (6) and state (7).

Step 6: Let $n=n-1$. If $n \geq 3$, go to Step 3. Otherwise, find the optimal value and corresponding sequence of $x$ to get the optimal solution.

\section{Basic Algorithm to UBQP5}

Then, in this section, we design the algorithm based on a different approach to solve $U B Q P 5$ where $Q$ is a fivediagonal symmetric matrix with zero diagonal elements which takes

$$
\left(\begin{array}{ccccccc}
0 & q_{12} & q_{13} & \cdots & 0 & 0 & 0 \\
q_{12} & 0 & q_{23} & \cdots & 0 & 0 & 0 \\
q_{13} & q_{23} & 0 & \cdots & 0 & 0 & 0 \\
\vdots & \vdots & \vdots & \ddots & \vdots & \vdots & \vdots \\
0 & 0 & 0 & \cdots & 0 & q_{n-2, n-1} & q_{n-2, n} \\
0 & 0 & 0 & \cdots & q_{n-2, n-1} & 0 & q_{n-1, n} \\
0 & 0 & 0 & \cdots & q_{n-2, n} & q_{n-1, n} & 0
\end{array}\right)
$$

Denote by $\Delta_{k}(x)$ the $k$ th derivative of $f(x)$ at $x$,

$$
\begin{aligned}
\Delta_{k}(x) & =\frac{\partial f(x)}{\partial x_{k}} \\
& =f\left(x_{1}, \ldots, x_{k-1}, 1, x_{k+1}, \ldots, x_{n}\right) \\
& -f\left(x_{1}, \ldots, x_{k-1}, 0, x_{k+1}, \ldots, x_{n}\right) .
\end{aligned}
$$

Denote by $\Theta_{k}(x)$ the $k$ th residual,

$$
\Theta_{k}(x)=f\left(x_{1}, \ldots, x_{k-1}, 0, x_{k+1}, \ldots, x_{n}\right)
$$

Then $f(x)$ can be expressed as

$$
f(x)=f_{k-1}(x)=x_{k} \Delta_{k}(x)+\Theta_{k}(x) .
$$

As is known from [11][12], a point $x \in\{0,1\}^{n}$ is a solution to $B Q P$ only if for all $k=1, \ldots, n$,

$$
x_{k}= \begin{cases}1, & \Delta_{\mathrm{k}}(\mathrm{x})<0, \\ 0, & \text { otherwise }\end{cases}
$$

The basic algorithm to $B Q P 5$ is developed based on the above necessary optimality condition. 
Through Equ.(2), we can readily get the following equation:

$$
\Delta_{k}\left(x_{k-2}, x_{k-1}\right)=q_{k-2, k} x_{k-2}+q_{k-1, k} x_{k-1}+c_{k} .
$$

So, we can express $f(x)$ in $U B Q P 5$ as

$$
\begin{aligned}
f(x) & =f_{k-1}(x) \\
& =x_{k} \Delta_{k}\left(x_{k-2}, x_{k-1}\right)+\Theta_{k}\left(x_{1}, \ldots, x_{k-1}\right) .
\end{aligned}
$$

Letting $s_{0}=c_{k}, s_{1}=q_{k-1, k}+c_{k}, s_{2}=q_{k-2, k}+c_{k}, s_{3}=q_{k-2, k}+q_{k-1, k}+c_{k}$. By taking advantage of the techniques in [11] and [12] and assigning different values to $s_{0}, s_{1}, s_{2}$ and $s_{3}$, we have constructed the expressions of $\Phi_{k}\left(x_{k-2}, x_{k-1}\right)$ and $\Phi_{k}\left(x_{k-2}, x_{k-1}\right) \Delta_{k}\left(x_{k-2}, x_{k-1}\right)$, see in Table 2. Here, $\Phi_{k}\left(x_{k-2}, x_{k-1}\right)$ is just the $x_{k}$ defined in Equ.(5). That is to say, $x_{k}=\Phi_{k}\left(x_{k-2}, x_{k-1}\right)$.

Table 2. Expressions of $\Phi_{k}\left(x_{k-2}, x_{k-1}\right)$ and $\Phi_{k}\left(x_{k-2}, x_{k-1}\right) \Delta_{k}\left(x_{k-2}, x_{k-1}\right)$

\begin{tabular}{|c|c|c|c|c|c|}
\hline$s_{0}$ & $s_{1}$ & $s_{2}$ & $s_{3}$ & $\Phi_{k}\left(x_{k-2}, x_{k-1}\right)$ & $\Phi_{k}\left(x_{k-2}, x_{k-1}\right) \Delta_{k}\left(x_{k-2}, x_{k-1}\right)$ \\
\hline$<0$ & $<0$ & $<0$ & $<0$ & 1 & $q_{k-2, k} x_{k-2}+q_{k-1, k} x_{k-1}+c_{k}$ \\
\hline$<0$ & $<0$ & $<0$ & $\geq 0$ & $1-x_{k-2} x_{k-1}$ & $-\left(q_{k-2, k}+q_{k-1, k}+c_{k}\right) x_{k-2} x_{k-1}+q_{k-2, k} x_{k-2}+q_{k-1, k} x_{k-1}+c_{k}$ \\
\hline$<0$ & $<0$ & $\geq 0$ & $<0$ & $1-x_{k-2}+x_{k-2} x_{k-1}$ & $\left(q_{k-2, k}+c_{k}\right) x_{k-2} x_{k-1}-c_{k} x_{k-2}+q_{k-1, k} x_{k-1}+c_{k}$ \\
\hline$<0$ & $<0$ & $\geq 0$ & $\geq 0$ & $1-x_{k-2}$ & $-q_{k-1, k} x_{k-2} x_{k-1}-c_{k} x_{k-2}+q_{k-1, k} x_{k-1}+c_{k}$ \\
\hline$<0$ & $\geq 0$ & $<0$ & $<0$ & $1-x_{k-1}+x_{k-2} x_{k-1}$ & $\left(q_{k-1, k}+c_{k}\right) x_{k-2} x_{k-1}+q_{k-2, k} x_{k-2}-c_{k} x_{k-1}+c_{k}$ \\
\hline$<0$ & $\geq 0$ & $<0$ & $\geq 0$ & $1-x_{k-1}$ & $-q_{k-2, k} x_{k-2} x_{k-1}+q_{k-2, k} x_{k-2}-c_{k} x_{k-1}+c_{k}$ \\
\hline$<0$ & $\geq 0$ & $\geq 0$ & $<0$ & $1-x_{k-2}-x_{k-1}+2 x_{k-2} x_{k-1}$ & $\left(q_{k-2, k}+q_{k-1, k}+2 c_{k}\right) x_{k-2} x_{k-1}-c_{k} x_{k-2}-c_{k} x_{k-1}+c_{k}$ \\
\hline$<0$ & $\geq 0$ & $\geq 0$ & $\geq 0$ & $1-x_{k-2}-x_{k-1}+x_{k-2} x_{k-1}$ & $c_{k} x_{k-2} x_{k-1}-c_{k} x_{k-2}-c_{k} x_{k-1}+c_{k}$ \\
\hline$\geq 0$ & $<0$ & $<0$ & $<0$ & $x_{k-2}+x_{k-1}-x_{k-2} x_{k-1}$ & $-c_{k} x_{k-2} x_{k-1}+\left(q_{k-2, k}+c_{k}\right) x_{k-2}+\left(q_{k-1, k}+c_{k}\right) x_{k-1}$ \\
\hline$\geq 0$ & $<0$ & $<0$ & $\geq 0$ & $x_{k-2}+x_{k-1}-2 x_{k-2} x_{k-1}$ & $-\left(q_{k-2, k}+q_{k-1, k}+2 c_{k}\right) x_{k-2} x_{k-1}+\left(q_{k-2, k}+c_{k}\right) x_{k-2}+\left(q_{k-1, k}+c_{k}\right) x_{k-1}$ \\
\hline$\geq 0$ & $<0$ & $\geq 0$ & $<0$ & $x_{k-1}$ & $q_{k-2, k} x_{k-2} x_{k-1}+\left(q_{k-1, k}+c_{k}\right) x_{k-1}$ \\
\hline$\geq 0$ & $<0$ & $\geq 0$ & $\geq 0$ & $x_{k-1}-x_{k-2} x_{k-1}$ & $-\left(q_{k-1, k}+c_{k}\right) x_{k-2} x_{k-1}+\left(q_{k-1, k}+c_{k}\right) x_{k-1}$ \\
\hline$\geq 0$ & $\geq 0$ & $<0$ & $<0$ & $x_{k-2}$ & $q_{k-1, k} x_{k-2} x_{k-1}+\left(q_{k-2, k}+c_{k}\right) x_{k-2}$ \\
\hline$\geq 0$ & $\geq 0$ & $<0$ & $\geq 0$ & $x_{k-2}-x_{k-2} x_{k-1}$ & $\left(q_{k-2, k}+c_{k}\right) x_{k-2} x_{k-1}+\left(q_{k-2, k}+c_{k}\right) x_{k-2}$ \\
\hline$\geq 0$ & $\geq 0$ & $\geq 0$ & $<0$ & $x_{k-2} x_{k-1}$ & $\left(q_{k-2, k}+q_{k-1, k}+c_{k}\right) x_{k-2} x_{k-1}$ \\
\hline$\geq 0$ & $\geq 0$ & $\geq 0$ & $\geq 0$ & 0 & 0 \\
\hline
\end{tabular}

Then, Equ.(7) can be rewritten to the form expressed in Equ.(8) by eliminating $x_{k}$.

$$
\begin{aligned}
f(x)= & f_{k-1}\left(x_{1}, \ldots, x_{k-1}\right) \\
= & \Phi_{k}\left(x_{k-2}, x_{k-1}\right) \Delta_{k}\left(x_{k-2}, x_{k-1}\right) \\
& +\Theta_{k}\left(x_{1}, \ldots, x_{k-1}\right) .
\end{aligned}
$$

By applying the same elimination process, we will get a series of equations $f_{k-1}\left(x_{1}, \ldots, x_{k-1}\right)(k=n, \ldots, 2)$. Finally, we can determine the optimal value of $U B Q P 5$.

Denote by $x^{*}$ the optimal solution of $U B Q P 5, x^{*}=\left\{x_{1}^{*}, x_{2}^{*}, \ldots, x_{n}^{*}\right\}$ can be easily obtained by utilizing the equation $x_{k}^{*}=\Phi_{k}\left(x_{k-2}^{*}, x_{k-1}^{*}\right)$ recursively for $k=1,2, \ldots n-1$.

Note that, in $U B Q P 5$, the expression $\Delta_{k}, \Phi_{k}$ and $\Phi_{k} \Delta_{k}$ are binomial functions of variable $x_{k-2}$ and $x_{k-1}$. Obviously, $\Theta_{k}$ is also a binomial expression of $x_{1}, \ldots, x_{k-1}$. Thus, $f_{k}$ remains a quadratic form throughout the iteration and the basic algorithm becomes polynomial[14].

Here, it should be noted that $\Phi_{k} \Delta_{k}$ can be described as $\Phi_{k} \Delta_{k}=a_{0} x_{k-2} x_{k-1}+b_{0} x_{k-2}+c_{0} x_{k-1}+d_{0} c_{k}$, which plays an important role in the polynomial time analysis.

Referring to Table 2, we can demonstrate the basic algorithm for $U B Q P 5$ as follows:

Algorithm 3.1. Basic Algorithm to UBQP5 
Step 1: $\quad$ Set $k=n$ and $f_{k}(x)=f(x)$.

Step 2: Calculate $\Delta_{k}\left(x_{k-2}, x_{k-1}\right)$ and $\Phi_{k}\left(x_{k-2}, x_{k-1}\right)$.

Step 3: Compute $f_{k}\left(x_{1}, \ldots, x_{k-1}\right)=\Phi_{k} \Delta_{k}+\Theta_{k}$.

Step 4: If $k>2$, then set $k=k-1$ and go to Step 2. Otherwise, $\operatorname{set}\left(x_{2}^{*}, x_{1}^{*}\right)=(0,0),(0,1),(1,0),(1,1)$ and determine the value of $f_{2}\left(x_{1}, x_{2}\right)$ respectively. And then, find out the optimal value and its corresponding solution $\left(x_{1}^{*}, x_{2}^{*}\right)$.

Step 5: $\quad$ Calculate $x_{k}^{*}$ by $x_{k}^{*}=\Phi_{k}\left(x_{k-2}^{*}, x_{k-1}^{*}\right)$ for $k=3, \ldots, n$.

For Algorithm 3.1, we can ensure that $f_{k}\left(x_{1}, \ldots, x_{k-1}\right)$ is also a quadratic function with $Q$ being a five-diagonal matrix, for $\Phi_{k} \Delta_{k}$ is always a binomial function of $x_{k-2}$ and $x_{k-1}$ at every iteration. Further more, $\Phi_{k} \Delta_{k}$ has been calculated according to Table 2 . As a result, this algorithm is very effective and efficient.

\section{Dynamic Programming Based Algorithm to LCBQP5}

We now, in this section, focus on solving $L C B Q P 5$ by combining Algorithm 3.1 and the dynamic programming method. The approach can also be applied to other linearly constrained binary quadratic programming problems.

First, we introduce a stage variable $k(0<k \leq n)$ and a state $s_{k}\left(s_{k} \in R\right)$ at stage $k$. And $s_{k}$ satisfies the following relationship

$$
s_{k+1}=s_{k}+a_{k} x_{k}, \quad k=1, \ldots, n-1,
$$

with $s_{1}=0$.

Since $a_{k}$ is integer-valued for $k=1, \ldots, n$, we just need to consider integer points in the state space (i.e. $s_{k} \in Z$ ). And we confine $s_{k}(k=2, \ldots, n)$ to $\underline{s}_{k} \leq s_{k} \leq \bar{s}_{k}$, where

$$
\begin{aligned}
\underline{s}_{k} & =\sum_{i=1}^{k-1} \min _{x_{i} \in\{0,1\}} a_{i} x_{i}=0, \\
\bar{s}_{k} & =\min \left\{\sum_{i=k}^{n} \max _{x_{i} \in\{0,1\}} a_{i} x_{i}, b-\sum_{i=k}^{n} \min _{x_{i} \in\{0,1\}} a_{i} x_{i}\right\} \\
& =\min \left\{\sum_{i=k}^{n} a_{i}, b\right\}
\end{aligned}
$$

Thus, for each stage $k$, we can easily define $\left\{s_{k}\right\}=\left\{\underline{s}_{k} \leq s_{k} \leq \bar{s}_{k}, s_{k} \in Z\right\}$ and the cost-to-go functions $\widehat{t}_{k}\left(s_{k}\right)$, a function defined over $\left\{x_{1}, \ldots, x_{k-1}\right\}$. See details in Procedure 4.1 and Procedure 4.2.

Procedure 4.1. Calculate the cost-to-go functions of $s_{k}$

Case 1: $k=n$

(1a) $s_{n}+a_{n}>b$

Obviously, $x_{n}$ must be set to 0 so as to satisfy the linear constraint. Thus, we can set $x_{n}^{*}=0$ and determine $\widehat{t_{n}}\left(s_{n}\right)$ by $\widehat{t_{n}}\left(s_{n}\right)=f\left(x_{1}, \ldots, x_{n-1}, 0\right)$.

(1b) $s_{n}+a_{n} \leq b$

In this case, it means $x_{n}$ can be either 0 or 1. That is, $x_{n} \in\{0,1\}$. By applying the first three steps of Algorithm 3.1 , we will obtain $x_{n}^{*}=\Phi_{n}\left(x_{n-2}, x_{n-1}\right)$ and $\widehat{t}_{n}\left(s_{n}\right)=f_{n-1}\left(x_{1}, \ldots, x_{n}\right)$.

Case 2: $k<n$

(2a) $s_{k}+a_{k} \notin s_{k+1}$

Similar to the described Case (1a), $x_{k}$ should be set to 0 (i.e. $x_{k}=0$ ). So, in this case, we just need to set $x_{k}^{*}=0$ and calculate $\widehat{t}_{k}\left(s_{k}\right)=\left.\widehat{t}_{k+1}\left(s_{k}\right)\right|_{x_{k}=0}$ (marked by SET_0). As to the details of calculation, please refer to Procedure 4.2 . 
(2b) $s_{k}+a_{k} \in s_{k+1}$

Also similar to the described Case (1b), $x_{k}$ can be either 0 or 1 (i.e. $\left.x_{n} \in\{0,1\}\right)$. And that, in this case, we ought to set $x_{k}^{*}=0$ in $\widehat{t}_{k+1}\left(s_{k}\right)$ and set $x_{k}=1$ in $\widehat{t}_{k+1}\left(s_{k}+a_{k}\right)$, and calculate the cost-to-go functions $\widehat{t}_{k}\left(s_{k}\right)$ respectively (the former marked by SET_0 and the latter SET_1). The procedure to $S E T_{-} 0$ here is the same as that in Case (2a). Refer to Procedure 4.2 for detail.

Obviously, for $k=n-1$, there is only one $\widehat{t}_{k+1}\left(s_{k}\right)$ and $\widehat{t}_{k+1}\left(s_{k}+a_{k}\right)$ for each state $s_{k}$. However, when $k<n-1$, there might exist multiple ones. Next, we should focus on finding out the optimal $\widehat{t_{k}}\left(s_{k}\right)$ in order to reduce the number of cost-to-go functions to the minimum. See Procedure 4.2 for more details. Here, we just demonstrate the procedure and then we will give the analysis and illustration in Section 5 and 6.

Procedure 4.2. Procedure to $S E T \_0$ and $S E T \_1$

SET_0

Case 1: If there is only one $\widehat{t}_{k+1}\left(s_{k}\right)$, set $x_{k}=0$ and $\widehat{t_{k}}\left(s_{k}\right)=\left.\widehat{t}_{k+1}\left(s_{k}\right)\right|_{x_{k}=0}$.

Case 2: If there are multiple $\widehat{t}_{k+1}\left(s_{k}\right)$, for the state $s_{k}$ in $\widehat{t}_{k+1}\left(s_{k}\right), x_{k+1}$ might be 0 or 1 . For both case, we find out respectively the $\widehat{t}_{k+1}\left(s_{k}\right)$ with the minimum constant term and then calculate $\widehat{t}_{k}\left(s_{k}\right)$ by setting $x_{k}=0$.

\section{SET_1}

Case 1: If there is only one $\widehat{t}_{k+1}\left(s_{k}\right)$, set $x_{k}=1$ and $\widehat{t_{k}}\left(s_{k}\right)=\left.\widehat{t}_{k+1}\left(s_{k}\right)\right|_{x_{k}=1}$.

Case 2: If there are multiple $\widehat{t}_{k+1}\left(s_{k}\right)$, for the state $s_{k}$ in $\widehat{t}_{k+1}\left(s_{k}+a_{k}\right), x_{k+1}$ might be 0 or 1 . For both case, we find out respectively the $\widehat{t}_{k+1}\left(s_{k}+a_{k}\right)$ with the minimum summation of the constant term and linear term coefficient of $x_{k}$, and then calculate $\widehat{t_{k}}\left(s_{k}\right)$ by setting $x_{k}=1$.

Through applying Procedure 4.1 and Procedure 4.2, we will generate a series of cost-to-go functions $\widehat{t}_{k}\left(s_{k}\right)$ with the property that they are only different in the linear term coefficient of $x_{k-2}, x_{k-1}$ and the constant coefficient except for $k=n$. When $k=n$, although the nonlinear coefficient of $x_{k-2} x_{k-1}$ is also different, it does not affect the calculation and polynomial feature of the algorithm. According to these two procedures above, we propose the following dynamic programming based algorithm.

\section{Algorithm 4.1. Dynamic Programming Based Algorithm to LCBQP5}

Step 1. Let $k=n$, for $s_{k}=0, \ldots, \bar{s}_{k}$, calculate $\widehat{t}_{k}\left(s_{k}\right)$ according to the procedure described Case 1 of Procedure 4.1.

Step 2. For $k=n-1, \ldots, 3$.

$$
\text { For } s_{k}=0, \ldots, \overline{s_{k}} \text {. }
$$

Calculate $\widehat{t}_{k}\left(s_{k}\right)$ according to Case 2 of Procedure 4.1.

Step 4. $\left(x_{2}^{*}, x_{1}^{*}\right):=(0,0),(0,1),(1,0),(1,1)$ and get the value of $s_{3}$ by the equation $s_{3}=a_{1} x_{1}+a_{2} x_{2}$. Then calculate the value of $\widehat{t_{3}}\left(s_{3}\right)$ respectively and find out the solution $\left(x_{1}^{*}, x_{2}^{*}\right)$ as well as its corresponding optimal value.

Step 5. Calculate the optimal solution by tracking, i.e., $x_{k}^{*}=\Phi_{k}\left(x_{k-2}^{*}, x_{k-1}^{*}\right), k=3, \ldots, n$.

In the previous procedures and algorithm, the maximum number of cost-to-go functions generated under different cases can be summarized in Table 3 .

From Table 3, we find out that there are at most four cost-to-go functions for each state $s_{k}$. For this reason, the number of cost-to-go functions generated at any stage $k$ will be less than four times that of states $s_{k}$ at this stage. The algorithm can be proved correct by applying the theorems stated in Chapter 7 of Li and Sun's book[5]. 
Table 3. Maximum number of cost-to-go functions for $s_{k}$

\begin{tabular}{|l|c|}
\hline Case in Procedure 4.1 & Maximum number of cost-to-go functions \\
\hline Case $(1 a),(1 b)$ & 1 \\
\hline Case $(2 a)$ & 2 \\
\hline Case $(2 b)$ & 4 \\
\hline
\end{tabular}

\section{Polynomial Time Solvable Property Analysis}

In this section, we analyze the polynomial time solvable property of the algorithms to $U B Q P 7, U B Q P 5$, and $L C B Q P 5$ we proposed.

We first analyze the algorithm to $U B Q P 7$. Since $Q$ is a seven-diagonal matrix, $f(x)$ takes the following form:

$$
\begin{aligned}
f(x)= & q_{12} x_{1} x_{2}+q_{13} x_{1} x_{3}+q_{14} x_{1} x_{4}+q_{23} x_{2} x_{3}+\ldots+q_{i-3, i} x_{i-3} x_{i}+q_{i-2, i} x_{i-2} x_{i} \\
& +q_{i-1, i} x_{i-1} x_{i}+\ldots+q_{n-1, n} x_{n-1} x_{n}+c_{1} x_{1}+\ldots+c_{i} x_{i}+\ldots+c_{n} x_{n}
\end{aligned}
$$

The terms related to $x_{i-3}, x_{i-2}, x_{i-1}$, and $x_{i}$ are $q_{i-3, i} x_{i-3} x_{i}, q_{i-2, i} x_{i-2} x_{i}, q_{i-1, i} x_{i-1} x_{i}, c_{i-3} x_{i-3}, c_{i-2} x_{i-2}, c_{i-1} x_{i-1}$, and $c_{i} x_{i}$. When the values of $x_{i-2}, x_{i-1}$, and $x_{i}$ are assigned. The coefficient of $x_{i-3}$ and constant in $f(x)$ will be $q_{i-3, i} x_{i}+c_{i-3}$ and $q_{i-2, i} x_{i-2} x_{i}+q_{i-1, i} x_{i-1} x_{i}+c_{i-2} x_{i-2}+c_{i-1} x_{i-1}+c_{i} x_{i}$ respectively. It is obvious that, for every two adjacent states of $x, f(x)$ are only different in the linear term coefficient of $x_{i-3}$ and the constant term. As a result, at each round of calculation, at most eight states will be generated based on the proposed algorithm and the computational complexity is proportional to $n$ approximately. Therefore the computational complexity of the Algorithm 2.1 is $O\left(n^{2}\right)$. The algorithm is polynomial time solvable.

Then we analyze the algorithm to UBQP5. Similar to Algorithm 2.1, Algorithm 3.1 consists of $n$ rounds of computation for an $n$ dimensional $U B Q P 5$. For a single round of computation, the computational computational complexity is approximately proportional to $n$. As a result, the computational complexity of Algorithm 3.1 is $O\left(n^{2}\right)$.

Finally, we make an analysis on the polynomial feature of the algorithm to $L C B Q P 5$. Actually, from Algorithm 4.1 and Table 3, we can easily get the conclusion that there are at most $4 \times(n-2) \times b$ cost-to-go functions for a n-dimensional problem.

For each calculation of a cost-to-go function, we just need to set the coefficients of $x_{k-2} x_{k}, x_{k-1} x_{k}$ and $x_{k}$ to zero and change the coefficients of $x_{k-2}, x_{k-1}$ and the constant term. Complementally, the coefficient of $x_{k-2} x_{k-1}$ should also be changed for $k=n$. It is reasonable, we can assume the computational complexity of each cost-to-go function calculation is proportional to $n$. Therefore, the computational complexity of Algorithm 4.1 is $O\left(b n^{3}\right)$. That is to say the complete algorithm takes polynomial time only. Later in Section 6 and Section 7, we will make an illustration.

Now, the main task is to analyze the rationality and correctness of Procedure 4.2. We make the analysis with the same flow path of Procedure 4.1. Assume $f(x)$ takes the following form:

$$
\begin{aligned}
f(x)= & q_{1,2} x_{1} x_{2}+\cdots+q_{n-2, n-1} x_{n-2} x_{n-1} \\
& +q_{n-2, n} x_{n-2} x_{n}+q_{n-1, n} x_{n-1} x_{n} \\
& +c_{1} x_{1}+\cdots+c_{n-2} x_{n-2}+c_{n-1} x_{n-1}+c_{n} x_{n} .
\end{aligned}
$$

Stage $_{-} \boldsymbol{k} \quad k=n$

Case_a $s_{n}+a_{n}>b$

$$
\begin{aligned}
\widehat{t_{n}}\left(s_{n}\right)= & f\left(x_{n}=0\right) \\
= & q_{1,2} x_{1} x_{2}+\cdots+q_{n-2, n-1} x_{n-2} x_{n-1} \\
& +c_{1} x_{1}+\cdots+c_{n-2} x_{n-2}+c_{n-1} x_{n-1} .
\end{aligned}
$$

Case_b $s_{n}+a_{n} \leq b$ 


$$
\begin{aligned}
\widehat{t}_{n}\left(s_{n}\right)= & f\left(x_{n}=0\right) \\
& +\Delta_{n}\left(x_{n-2}, x_{n-1}\right) \Phi_{n}\left(x_{n-2}, x_{n-1}\right) \\
= & f\left(x_{n}=0\right)+a_{0} x_{n-2} x_{n-1} \\
& +b_{0} x_{n-2}+c_{0} x_{n-1}+d_{0} c_{n} \\
= & q_{1,2} x_{1} x_{2}+\ldots \\
& +\left(q_{n-2, n-1}+a_{0}\right) x_{n-2} x_{n-1} \\
& +c_{1} x_{1}+\cdots+\left(c_{n-2}+b_{0}\right) x_{n-2} \\
& +\left(c_{n-1}+c_{0}\right) x_{n-1}+d_{0} c_{n} .
\end{aligned}
$$

In this stage, each state $s_{k}$ only has one $\widehat{t_{k}}\left(s_{k}\right)$. Obviously, all $\widehat{t_{n}}\left(s_{n}\right)$ have the same coefficients of nonlinear and linear terms except for that of $x_{n-2} x_{n-1}, x_{n-2}, x_{n-1}$ and the constant term.

\section{Stage_(n-1) $\quad k=n-1$}

Case $a s_{k}+a_{k} \notin s_{k+1}$. Perform SET_0.

For Stage_n, only one $\widehat{t_{k}}+1\left(s_{k}\right)$ exists for each state $s_{n}$. If $s_{n}+a_{n}>b$, by Case_a in Stage_n, we get

$$
\begin{aligned}
\widehat{t_{k}}\left(s_{k}\right)= & q_{1,2} x_{1} x_{2}+\cdots+q_{k-3, k-1} x_{k-3} x_{k-1} \\
& +q_{k-2, k-1} x_{k-2} x_{k-1} \\
& +c_{1} x_{1}+\cdots+c_{k-2} x_{k-2}+c_{k_{1}} x_{k-1} .
\end{aligned}
$$

If $s_{n}+a_{n} \leq b$, by Case_b in Stage_n, we get

$$
\begin{aligned}
\widehat{t_{k}}\left(s_{k}\right)= & q_{1,2} x_{1} x_{2}+\cdots+q_{k-3, k-1} x_{k-3} x_{k-1} \\
& +q_{k-2, k-1} x_{k-2} x_{k-1} \\
& +c_{1} x_{1}+\cdots+c_{k-2} x_{k-2} \\
& +\left(c_{k-1}+b_{0}\right) x_{k-1}+d_{0} c_{n} .
\end{aligned}
$$

In this case, each state $s_{k}$ only has one $\widehat{t_{k}}\left(s_{k}\right)$.

Case $\boldsymbol{b} \quad s_{k}+a_{k} \in s_{k+1}$. Perform $S E T \_0$ and $S E T_{-} 1$.

Here, the procedure $S E T \_0$ is the same with Case_a in Stage_(n-1). We just need to consider $S E T_{-} 1$. If $s_{n}+a_{n}>b$, by Case_b in Stage_n, we get

$$
\begin{aligned}
\widehat{t_{k}}\left(s_{k}\right)= & q_{1,2} x_{1} x_{2}+\cdots+q_{k-3, k-1} x_{k-3} x_{k-1} \\
& +q_{k-2, k-1} x_{k-2} x_{k-1} \\
& +c_{1} x_{1}+\cdots+\left(q_{k-2, k}+c_{k-2}\right) x_{k-2} \\
& +\left(q_{k-1, k}+c_{k-1}\right) x_{k-1}+c_{k} .
\end{aligned}
$$

If $s_{n}+a_{n} \leq b$, by Case_b in Stage_n, we get

$$
\begin{aligned}
\widehat{t_{k}}\left(s_{k}\right)= & q_{1,2} x_{1} x_{2}+\cdots+q_{k-3, k-1} x_{k-3} x_{k-1} \\
& +q_{k-2, k-1} x_{k-2} x_{k-1} \\
& +c_{1} x_{1}+\cdots+\left(q_{k-2, k}+c_{k-2}\right) x_{k-2} \\
& +\left(q_{k-1, k}+c_{k-1}+b_{0}\right) x_{k-1} \\
& +\left(c_{k}+c_{0}+d_{0} c_{n}\right) .
\end{aligned}
$$

In this case, each state $s_{k}$ has two $\widehat{t_{k}}\left(s_{k}\right)$. 
In this stage, each state $s_{k}$ has at most two $\widehat{t_{k}}\left(s_{k}\right)$. Obviously, all $\widehat{t_{k}}\left(s_{k}\right)$ have the same coefficients of nonlinear and linear terms except for that of $x_{k-2}, x_{k-1}$ and the constant term. We describe all these $\widehat{t}_{k}\left(s_{k}\right)$ as

$$
\begin{aligned}
\widehat{t}_{k}\left(s_{k}\right)= & q_{1,2} x_{1} x_{2}+\cdots+q_{k-3, k-1} x_{k-3} x_{k-1} \\
& +q_{k-2, k-1} x_{k-2} x_{k-1}+c_{1} x_{1}+\ldots \\
& +a_{0} x_{k-2}+b_{0} x_{k-1}+c_{0} .
\end{aligned}
$$

Stage_(n-2) $\quad k=n-2$

Similar to Stage_(n-1), all $\widehat{t}_{k}\left(s_{k}\right)$ can be generated with the same form as Equ.(10). Now we just consider the difference in each state $s_{k}$ and extremely assume that each of $\widehat{t}_{k+1}\left(s_{k}\right)$ and $\widehat{t}_{k+1}\left(s_{k}+a_{k}\right)$ are double (i.e., $x_{k+1}=0$ or 1$)$. First perform $S E T \_0$ and then perform $S E T T_{-} 1$.

Case_a Perform SET_0.

Here we should do with $\widehat{t}_{k+1}\left(s_{k}\right)$ described in Equ.(10).

$$
\begin{aligned}
\widehat{t}_{k}\left(s_{k}\right) & =\left.\widehat{t}_{k+1}\left(s_{k}\right)\right|_{x_{k}=0} \\
& =q_{1,2} x_{1} x_{2}+\cdots+q_{k-2, k-1} x_{k-2} x_{k-1} \\
& +c_{1} x_{1}+\cdots+c_{k-2} x_{k-2} \\
& +a_{0}^{\prime} x_{k-1}+c_{0}^{\prime} .
\end{aligned}
$$

Case $b$ Perform $S E T \_1$.

Here we should do with $\widehat{t}_{k+1}\left(s_{k}+a_{k}\right)$ described in Equ.(10).

$$
\begin{aligned}
\widehat{t}_{k}\left(s_{k}\right) & =\left.\widehat{t}_{k+1}\left(s_{k}+a_{k}\right)\right|_{x_{k}=1} \\
& =q_{1,2} x_{1} x_{2}+\cdots+q_{k-2, k-1} x_{k-2} x_{k-1} \\
& +c_{1} x_{1}+\cdots+\left(q_{k-2, k}+c_{k-2}\right) x_{k-2} \\
& +\left(q_{k-1, k}+a_{0}^{\prime}\right) x_{k-1}+c_{0}^{\prime} .
\end{aligned}
$$

In this stage, each state $s_{k}$ has at most four $\widehat{t_{k}}\left(s_{k}\right)$. Note that, both Equ.(11) and Equ.(12) include two subexpressions varying with $a_{0}^{\prime}$ and $c_{0}^{\prime}$. That is say, they are different from the constant term and the coefficient of linear term $x_{k-1}$. Obviously, for the next step, by performing $S E T \_0$ and $S E T_{-} 1$, we can find out a better solution for each different cases in Procedure 4.2, which has demonstrated the rationality of this procedure.

Similar to the above process, for every remaining stage $k$, all $\widehat{t_{k}}\left(s_{k}\right)$ can be generated with the same form as Equ.(10) and each state $s_{k}$ has at most four $\widehat{t}_{k}\left(s_{k}\right)$. And for Case 2 in Procedure 4.2, the only differences between the multiple expressions are the constant term and the linear coefficient of $x_{k-1}$. We can just compare the summation of the constant term and the linear coefficient of $x_{k-1}$ to perform $S E T_{-} 1$ and compare the constant term to perform $S E T \_0$. Thus, Procedure 4.2 is reasonable as well as correct and can lead to a limited size of calculation, which finally makes Algorithm 4.1 effective and efficient.

\section{Examples}

In this section, we apply the above proposed algorithms to solve the examples of $B Q P 7, B Q P 5$ and $L C B Q P 5$ respectively to check their effectiveness.

First, we apply the algorithm proposed in Section 2 to solve the following $B Q P 7$ example by perform Procedure. 2.1 to 2.3 step by step.

\section{Example 6.1.}

$$
\begin{aligned}
\min _{x \in\{0,1\}^{6}} f(x) & =23 x_{1} x_{2}-37 x_{1} x_{3}-56 x_{1} x_{4}+41 x_{2} x_{3}+16 x_{2} x_{4}-34 x_{2} x_{5}-62 x_{3} x_{4}-27 x_{3} x_{5} \\
& +76 x_{3} x_{6}-81 x_{4} x_{5}+14 x_{4} x_{6}-58 x_{4} x_{7}+90 x_{5} x_{6}+25 x_{5} x_{7}-42 x_{5} x_{8}-12 x_{6} x_{7} \\
& +31 x_{6} x_{8}-94 x_{7} x_{8}+24 x_{1}-54 x_{2}-17 x_{3}+36 x_{4}-72 x_{5}+63 x_{6}+46 x_{7}-18 x_{8}
\end{aligned}
$$


Table 4. Values of $f(x)$ when $x_{6} \sim x_{8}$ being set

\begin{tabular}{|c|l|l|}
\hline$x_{6}$ & $\left(x_{6} x_{7} x_{8}\right)$ & the expression of $f(x)$ \\
\hline 0 & $(0,0,0)$ & $\begin{array}{l}23 x_{1} x_{2}-37 x_{1} x_{3}-56 x_{1} x_{4}+41 x_{2} x_{3}+16 x_{2} x_{4}-34 x_{2} x_{5}-62 x_{3} x_{4} \\
-27 x_{3} x_{5}-81 x_{4} x_{5}+24 x_{1}-54 x_{2}-17 x_{3}+36 x_{4}-\mathbf{7 2} x_{5}\end{array}$ \\
\hline 0 & $(0,0,1)$ & $\begin{array}{l}23 x_{1} x_{2}-37 x_{1} x_{3}-56 x_{1} x_{4}+41 x_{2} x_{3}+16 x_{2} x_{4}-34 x_{2} x_{5}-62 x_{3} x_{4} \\
-27 x_{3} x_{5}-81 x_{4} x_{5}+24 x_{1}-54 x_{2}-17 x_{3}+36 x_{4}-\mathbf{1 1 4} x_{5}-\mathbf{1 8}\end{array}$ \\
\hline 0 & $(0,1,0)$ & $\begin{array}{l}23 x_{1} x_{2}-37 x_{1} x_{3}-56 x_{1} x_{4}+41 x_{2} x_{3}+16 x_{2} x_{4}-34 x_{2} x_{5}-62 x_{3} x_{4} \\
-27 x_{3} x_{5}-81 x_{4} x_{5}+24 x_{1}-54 x_{2}-17 x_{3}-22 x_{4}-\mathbf{4 7} x_{5}+46\end{array}$ \\
\hline 0 & $(0,1,1)$ & $\begin{array}{l}23 x_{1} x_{2}-37 x_{1} x_{3}-56 x_{1} x_{4}+41 x_{2} x_{3}+16 x_{2} x_{4}-34 x_{2} x_{5}-62 x_{3} x_{4} \\
-27 x_{3} x_{5}-81 x_{4} x_{5}+24 x_{1}-54 x_{2}-17 x_{3}-22 x_{4}-\mathbf{8 9} x_{5}-\mathbf{6 6}\end{array}$ \\
\hline 1 & $(1,0,0)$ & $\begin{array}{l}23 x_{1} x_{2}-37 x_{1} x_{3}-56 x_{1} x_{4}+41 x_{2} x_{3}+16 x_{2} x_{4}-34 x_{2} x_{5}-62 x_{3} x_{4} \\
-27 x_{3} x_{5}-81 x_{4} x_{5}+24 x_{1}-54 x_{2}+59 x_{3}+50 x_{4}+\mathbf{1 8} x_{5}+\mathbf{6 3}\end{array}$ \\
\hline 1 & $(1,0,1)$ & $\begin{array}{l}23 x_{1} x_{2}-37 x_{1} x_{3}-56 x_{1} x_{4}+41 x_{2} x_{3}+16 x_{2} x_{4}-34 x_{2} x_{5}-62 x_{3} x_{4} \\
-27 x_{3} x_{5}-81 x_{4} x_{5}+24 x_{1}-54 x_{2}+59 x_{3}+50 x_{4}-\mathbf{2 4} x_{5}+\mathbf{7 6}\end{array}$ \\
\hline 1 & $(1,1,0)$ & $\begin{array}{l}23 x_{1} x_{2}-37 x_{1} x_{3}-56 x_{1} x_{4}+41 x_{2} x_{3}+16 x_{2} x_{4}-34 x_{2} x_{5}-62 x_{3} x_{4} \\
-27 x_{3} x_{5}-81 x_{4} x_{5}+24 x_{1}-54 x_{2}+59 x_{3}-8 x_{4}+\mathbf{4 3} x_{5}+\mathbf{9 7}\end{array}$ \\
\hline 1 & $(1,1,1)$ & $\begin{array}{l}23 x_{1} x_{2}-37 x_{1} x_{3}-56 x_{1} x_{4}+41 x_{2} x_{3}+16 x_{2} x_{4}-34 x_{2} x_{5}-62 x_{3} x_{4} \\
-27 x_{3} x_{5}-81 x_{4} x_{5}+24 x_{1}-54 x_{2}+59 x_{3}-8 x_{4}+\mathbf{1} x_{5}+\mathbf{1 6}\end{array}$ \\
\hline
\end{tabular}

First we set $x_{8}, x_{7}$ and $x_{6}$ to 0 or 1 , obtaining eight states of $f(x)$ in Table 4 . For each two adjacent states (only $x_{8}$ is different), only the last two terms are different. For state $(0,0,0)$ and $(0,0,1)$, the different terms are $\left(-72 x_{5}\right)$ and $\left(-114 x_{5}-18\right)$. Therefore, by setting $x_{5}=0$ or $x_{5}=1$, compare the two states and choose an optimal one. Repeat this procedure for state $(0,1,0)$ and $(0,1,1),(1,0,0)$ and $(1,0,1),(1,1,0)$ and $(1,1,1)$, we can generate Table 5.

Table 5. Values of $f(x)$ after choosing the better solution

\begin{tabular}{|c|l|l|}
\hline$x_{5}$ & $\left(x_{5} x_{6} x_{7} x_{8}\right)$ & the expression of $f(x)$ \\
\hline 0 & $(0,0,0,1)$ & $23 x_{1} x_{2}-37 x_{1} x_{3}-56 x_{1} x_{4}+41 x_{2} x_{3}+16 x_{2} x_{4}-62 x_{3} x_{4}+24 x_{1}-54 x_{2}-17 x_{3}+\mathbf{3 6} x_{4}-\mathbf{1 8}$ \\
\hline 0 & $(0,0,1,1)$ & $23 x_{1} x_{2}-37 x_{1} x_{3}-56 x_{1} x_{4}+41 x_{2} x_{3}+16 x_{2} x_{4}-62 x_{3} x_{4}+24 x_{1}-54 x_{2}-17 x_{3}-\mathbf{2 2} x_{4}-\mathbf{6 6}$ \\
\hline 0 & $(0,1,0,0)$ & $23 x_{1} x_{2}-37 x_{1} x_{3}-56 x_{1} x_{4}+41 x_{2} x_{3}+16 x_{2} x_{4}-62 x_{3} x_{4}+24 x_{1}-54 x_{2}+59 x_{3}+\mathbf{4 0} x_{4}+\mathbf{6 3}$ \\
\hline 0 & $(0,1,1,1)$ & $23 x_{1} x_{2}-37 x_{1} x_{3}-56 x_{1} x_{4}+41 x_{2} x_{3}+16 x_{2} x_{4}-62 x_{3} x_{4}+24 x_{1}-54 x_{2}+59 x_{3}-\mathbf{8} x_{4}+\mathbf{1 6}$ \\
\hline 1 & $(1,0,0,1)$ & $23 x_{1} x_{2}-37 x_{1} x_{3}-56 x_{1} x_{4}+41 x_{2} x_{3}+16 x_{2} x_{4}-62 x_{3} x_{4}+24 x_{1}-88 x_{2}-44 x_{3}-\mathbf{4 5} x_{4}-\mathbf{1 3 2}$ \\
\hline 1 & $(1,0,1,1)$ & $23 x_{1} x_{2}-37 x_{1} x_{3}-56 x_{1} x_{4}+41 x_{2} x_{3}+16 x_{2} x_{4}-62 x_{3} x_{4}+24 x_{1}-88 x_{2}-44 x_{3}-\mathbf{1 0 3} x_{4}+\mathbf{5 2}$ \\
\hline 1 & $(1,1,0,1)$ & $23 x_{1} x_{2}-37 x_{1} x_{3}-56 x_{1} x_{4}+41 x_{2} x_{3}+16 x_{2} x_{4}-62 x_{3} x_{4}+24 x_{1}-88 x_{2}+32 x_{3}-\mathbf{3 1} x_{4}+\mathbf{7 6}$ \\
\hline 1 & $(1,1,1,1)$ & $23 x_{1} x_{2}-37 x_{1} x_{3}-56 x_{1} x_{4}+41 x_{2} x_{3}+16 x_{2} x_{4}-62 x_{3} x_{4}+24 x_{1}-88 x_{2}+32 x_{3}-\mathbf{8 9} x_{4}+\mathbf{1 7}$ \\
\hline
\end{tabular}

Repeating this step, we can get the similar tables by setting $x_{4}$ and $x_{3}$ respectively. Then, we set $x_{2}$ to 0 or 1 and get Table 6

In Table 6, the values of the first two forms of $f(x)$ are: -155 and -258 when we set $x_{i}$ to 0 . The smaller one -258 is remained. And, for the next two states, the value -364 is kept. Repeat this procedure for the remaining data, we finally obtain the Table 7.

We can see from Table 7 that the minimum value of $f(x)$ is -441 , the corresponding optimal solution is $x=$ $(1,1,1,1,1,0,1,1)$.

Then, for $U B Q P 5$ problem, we apply Algorithm 3.1 to the following example: 
Table 6. Values of $f(x)$ and its corresponding solution

\begin{tabular}{|l|c|l|}
\hline$x_{2}$ & $\left(x_{2} \ldots x_{8}\right)$ & the expression of $f(x)$ \\
\hline 0 & $(0,0,0,1,0,1,1)$ & $\mathbf{2 4} x_{1} \mathbf{- 1 5 5}$ \\
\hline 0 & $(0,0,1,1,0,1,1)$ & $\mathbf{- 3 2} x_{1} \mathbf{- 2 5 8}$ \\
\hline 0 & $(0,1,0,1,0,1,1)$ & $\mathbf{- 1 3} x_{1} \mathbf{- 1 9 9}$ \\
\hline 0 & $(0,1,1,1,0,1,1)$ & $\mathbf{- 6 9} x_{1} \mathbf{- 3 6 4}$ \\
\hline 1 & $(1,0,0,1,0,1,1)$ & $\mathbf{4 7} x_{1} \mathbf{- 2 4 3}$ \\
\hline 1 & $(1,0,1,1,0,1,1)$ & $\mathbf{- 9} x_{1} \mathbf{- 3 3 0}$ \\
\hline 1 & $(1,1,0,1,0,1,1)$ & $\mathbf{1 0} x_{1} \mathbf{- 2 4 6}$ \\
\hline 1 & $(1,1,1,1,0,1,1)$ & $\mathbf{- 4 6} x_{1} \mathbf{- 3 9 5}$ \\
\hline
\end{tabular}

Table 7. Solutions and the corresponding values

\begin{tabular}{|c|c|l|}
\hline$x_{1}$ & $\left(x_{1} \ldots x_{8}\right)$ & the values of $f(x)$ \\
\hline 0 & $(0,0,0,1,1,0,1,1)$ & $\mathbf{- 2 5 8}$ \\
\hline 0 & $(0,0,1,1,1,0,1,1)$ & $\mathbf{- 3 6 4}$ \\
\hline 0 & $(0,1,0,1,1,0,1,1)$ & $\mathbf{- 3 3 0}$ \\
\hline 0 & $(0,1,1,1,1,0,1,1)$ & $\mathbf{- 3 9 5}$ \\
\hline 1 & $(1,0,0,1,1,0,1,1)$ & $\mathbf{- 2 9 0}$ \\
\hline 1 & $(1,0,1,1,1,0,1,1)$ & $\mathbf{- 4 3 3}$ \\
\hline 1 & $(1,1,0,1,1,0,1,1)$ & $\mathbf{- 3 3 9}$ \\
\hline 1 & $(1,1,1,1,1,0,1,1)$ & $\mathbf{- 4 4 1}$ \\
\hline
\end{tabular}

\section{Example 6.2.}

$$
\begin{gathered}
\min _{x \in\{0,1\}^{6}} f(x)=36 x_{1} x_{2}-22 x_{1} x_{3}-34 x_{2} x_{3}+46 x_{2} x_{4}+64 x_{3} x_{4}-92 x_{3} x_{5} \\
+46 x_{4} x_{5}-16 x_{4} x_{6}+50 x_{5} x_{6}+16 x_{1}-22 x_{2} \\
+36 x_{3}-48 x_{4}+56 x_{5}-20 x_{6}
\end{gathered}
$$
Then,

$$
\begin{aligned}
& f_{4}=36 x_{1} x_{2}-22 x_{1} x_{3}-34 x_{2} x_{3}+46 x_{2} x_{4}+48 x_{3} x_{4} \\
& +16 x_{1}-22 x_{2}+36 x_{3}-64 x_{4}-20
\end{aligned}
$$

$k=4, \Delta_{4}=46 x_{2}+48 x_{3}-64, s_{0}=-64<0, s_{1}=-16<0, s_{2}=-18<0, s_{3}=30>0, x_{4}{ }^{*}=\Phi_{4}=1-x_{2} x_{3}$. Then,

$$
\begin{gathered}
f_{3}=36 x_{1} x_{2}-22 x_{1} x_{3}-64 x_{2} x_{3}+16 x_{1}-22 x_{2}+68 x_{3}-84 \\
k=3, \Delta_{3}=-22 x_{1}-64 x_{2}+68, s_{0}=68>0, s_{1}=4>0, s_{2}=46>0, s_{3}=-18<0, x_{3}{ }^{*}=\Phi_{3}=x_{1} x_{2} . \text { Then, } \\
f_{2}=18 x_{1} x_{2}+16 x_{1}+24 x_{2}-84 \\
k=1, f_{2}(0,0)=-84, f_{2}(0,1)=-60, f_{2}(1,0)=-68, f_{2}(1,1)=-26 .
\end{gathered}
$$


Thus, $\min _{x \in\{0,1\}^{5}} f(x)=-84, x_{1}^{*}=0, x_{2}^{*}=0 . x_{3}{ }^{*}=\Phi_{3}=x_{1} x_{2}=0, x_{4}{ }^{*}=\Phi_{4}=1-x_{2} x_{3}=1, x_{5}{ }^{*}=\Phi_{5}=x_{3}-x_{3} x_{4}=$ $0 £ x_{6}{ }^{*}=\Phi_{6}=1-x_{5}=1$.

As a result, $x^{*}=(0,0,0,1,0,1)^{T}, f\left(x^{*}\right)=-84$.

Finally, for $L C B Q P 5$ problem, we illustrate Algorithm 4.1 and verify the analysis in Section 5 by solving the following example.

\section{Example 6.3.}

$$
\begin{aligned}
\min _{x \in\{0,1\}^{6}} f(x) & =36 x_{1} x_{2}-22 x_{1} x_{3}-34 x_{2} x_{3} \\
& +46 x_{2} x_{4}-92 x_{3} x_{5}+46 x_{4} x_{5} \\
& -16 x_{4} x_{6}+50 x_{5} x_{6}+16 x_{1}-22 x_{2} \\
& +36 x_{3}-48 x_{4}+56 x_{5}-20 x_{6}, \\
\text { s.t. } \quad 3 x_{1} & +2 x_{2}+4 x_{3}+3 x_{4}+4 x_{5}+5 x_{6} \leq 6 .
\end{aligned}
$$

With the enumeration method, we get 12 solutions satisfying the linear constraint. The optimal solution is $x^{*}=$ $(0,0,0,1,0,0)$ with the corresponding value $f\left(x^{*}\right)=-48$.

Now, we apply Algorithm 4.1 to this example.

First, we generate the table for $s_{6}$. For this stage, the table should be constructed according to Case 1 in Procedure 4.1. Refer to Table 8. Here, $s_{6}=a_{1} x_{1}+\cdots+a_{5} x_{5}, s_{6}+a_{6}=s_{6}+5, b=6, s_{6}=\{0, \ldots, 6\}$.

Table 8. Cost-to-go functions for $\widehat{t_{6}}\left(s_{6}\right)$

\begin{tabular}{|c|c|c|l|}
\hline$s_{6}$ & $x_{6}$ & $x_{6}^{*}$ & $\widehat{t}_{6}\left(s_{6}\right)$ \\
\hline 0 & $\{0,1\}$ & $1-x_{5}$ & $\begin{array}{l}36 x_{1} x_{2}-22 x_{1} x_{3}-34 x_{2} x_{3}+46 x_{2} x_{4} \\
+64 x_{3} x_{4}-92 x_{3} x_{5}+\mathbf{6 2} x_{4} x_{5} \\
+16 x_{1}-22 x_{2}+36 x_{3}-\mathbf{6 4} x_{4}+\mathbf{7 6} x_{5}-\mathbf{2 0}\end{array}$ \\
\hline 1 & $\{0,1\}$ & $1-x_{5}$ & $\begin{array}{l}36 x_{1} x_{2}-22 x_{1} x_{3}-34 x_{2} x_{3}+46 x_{2} x_{4} \\
+64 x_{3} x_{4}-92 x_{3} x_{5}+\mathbf{6 2} x_{4} x_{5} \\
+16 x_{1}-22 x_{2}+36 x_{3}-\mathbf{6 4} x_{4}+\mathbf{7 6} x_{5}-\mathbf{2 0}\end{array}$ \\
\hline 2 & 0 & 0 & $\begin{array}{l}36 x_{1} x_{2}-22 x_{1} x_{3}-34 x_{2} x_{3}+46 x_{2} x_{4} \\
+64 x_{3} x_{4}-92 x_{3} x_{5}+\mathbf{4 6} x_{4} x_{5} \\
+16 x_{1}-22 x_{2}+36 x_{3}-\mathbf{4 8} x_{4}+\mathbf{5 6} x_{5}+\mathbf{0}\end{array}$ \\
\hline 3 & 0 & 0 & $\begin{array}{l}36 x_{1} x_{2}-22 x_{1} x_{3}-34 x_{2} x_{3}+46 x_{2} x_{4} \\
+64 x_{3} x_{4}-92 x_{3} x_{5}+\mathbf{4 6} x_{4} x_{5} \\
+16 x_{1}-22 x_{2}+36 x_{3}-\mathbf{4 8} x_{4}+\mathbf{5 6} x_{5}+\mathbf{0}\end{array}$ \\
\hline 4 & 0 & 0 & $\begin{array}{l}36 x_{1} x_{2}-22 x_{1} x_{3}-34 x_{2} x_{3}+46 x_{2} x_{4} \\
+64 x_{3} x_{4}-92 x_{3} x_{5}+\mathbf{4 6} x_{4} x_{5} \\
+16 x_{1}-22 x_{2}+36 x_{3}-\mathbf{4 8} x_{4}+\mathbf{5 6} x_{5}+\mathbf{0}\end{array}$ \\
\hline 5 & 0 & 0 & $\begin{array}{l}36 x_{1} x_{2}-22 x_{1} x_{3}-34 x_{2} x_{3}+46 x_{2} x_{4} \\
+64 x_{3} x_{4}-92 x_{3} x_{5}+\mathbf{4 6} x_{4} x_{5} \\
+16 x_{1}-22 x_{2}+36 x_{3}-\mathbf{4 8} x_{4}+\mathbf{5 6} x_{5}+\mathbf{0}\end{array}$ \\
\hline 6 & 0 & 0 & $\begin{array}{l}36 x_{1} x_{2}-22 x_{1} x_{3}-34 x_{2} x_{3}+46 x_{2} x_{4} \\
+64 x_{3} x_{4}-92 x_{3} x_{5}+\mathbf{4 6} x_{4} x_{5} \\
+16 x_{1}-22 x_{2}+36 x_{3}-\mathbf{4 8} x_{4}+\mathbf{5 6} x_{5}+\mathbf{0}\end{array}$ \\
\hline
\end{tabular}

In Table 8, there are seven possible states, i.e., $s_{6}=0, \ldots, 6$. For $s_{6}=0,1, s_{6}+5 \leq 6$ is satisfied, we get $x_{6}^{*}$ and $\widehat{t}_{6}\left(s_{6}\right)$ from Case (1b) in Procedure 4.1. And for others, we get that from Case (1a). Note that, as described in Section 5 , all $\widehat{t}_{6}\left(s_{6}\right)$ are only different from the coefficients of $x_{4} x_{5}, x_{4}, x_{5}$ and the constant term. 
In the following steps, since $k=5,4,3, k<n=6$, the remaining tables should be constructed according to Case 2 in Procedure 4.1.

Here, we skip the tables for $s_{5}$ and $s_{4}$ and only present the table for $s_{3}$ as shown in Table 9 .

Table 9. Cost-to-go functions for $\widehat{t_{3}}\left(s_{3}\right)$

\begin{tabular}{|c|c|c|l|}
\hline$s_{3}$ & $x_{3}$ & $x_{3}^{*}$ & $\widehat{t}_{3}\left(s_{3}\right)$ \\
\hline 0 & $\{0,1\}$ & 0 & $\left.\widehat{t}_{4}(0)\right|_{x_{3}=0}=36 x_{1} x_{2}+\mathbf{1 6} x_{1}-\mathbf{2 2} x_{2} \mathbf{- 2 0}$ \\
\hline 0 & $\{0,1\}$ & 0 & $\left.\widehat{t}_{4}(0)\right|_{x_{3}=0}=36 x_{1} x_{2}+\mathbf{1 6} x_{1}+\mathbf{2 4} x_{2}-\mathbf{4 8}$ \\
\hline 0 & $\{0,1\}$ & 1 & $\left.\widehat{t_{4}}(4)\right|_{x_{3}=1}=36 x_{1} x_{2}-\mathbf{6} x_{1}-\mathbf{5 6} x_{2}+\mathbf{3 6}$ \\
\hline 1 & $\{0,1\}$ & 0 & $\left.\widehat{t}_{4}(1)\right|_{x_{3}=0}=36 x_{1} x_{2}+\mathbf{1 6} x_{1}-\mathbf{2 2} x_{2}-\mathbf{2 0}$ \\
\hline 1 & $\{0,1\}$ & 0 & $\left.\widehat{t}_{4}(1)\right|_{x_{3}=0}=36 x_{1} x_{2}+\mathbf{1 6} x_{1}+\mathbf{2 4} x_{2}-\mathbf{4 8}$ \\
\hline 1 & $\{0,1\}$ & 1 & $\left.\widehat{t}_{4}(5)\right|_{x_{3}=1}=36 x_{1} x_{2}-\mathbf{6} x_{1}-\mathbf{5 6} x_{2}+\mathbf{3 6}$ \\
\hline 2 & $\{0,1\}$ & 0 & $\left.\widehat{t}_{4}(2)\right|_{x_{3}=0}=36 x_{1} x_{2}+\mathbf{1 6} x_{1}-\mathbf{2 2} x_{2}+\mathbf{0}$ \\
\hline 2 & $\{0,1\}$ & 0 & $\left.\widehat{t}_{4}(2)\right|_{x_{3}=0}=36 x_{1} x_{2}+\mathbf{1 6} x_{1}+\mathbf{2 4} x_{2}-\mathbf{4 8}$ \\
\hline 2 & $\{0,1\}$ & 1 & $\left.\widehat{t}_{4}(6)\right|_{x_{3}=1}=36 x_{1} x_{2}-\mathbf{6} x_{1}-\mathbf{5 6} x_{2}+\mathbf{3 6}$ \\
\hline 3 & 0 & 0 & $\left.\widehat{t}_{4}(3)\right|_{x_{3}=0}=36 x_{1} x_{2}+\mathbf{1 6} x_{1}-\mathbf{2 2} x_{2}+\mathbf{0}$ \\
\hline 3 & 0 & 0 & $\left.\widehat{t}_{4}(3)\right|_{x_{3}=0}=36 x_{1} x_{2}+\mathbf{1 6} x_{1}+\mathbf{2 4} x_{2}-\mathbf{4 8}$ \\
\hline 4 & 0 & 0 & $\left.\widehat{t}_{4}(4)\right|_{x_{3}=0}=36 x_{1} x_{2}+\mathbf{1 6} x_{1} \mathbf{- 2 2} x_{2}+\mathbf{0}$ \\
\hline 5 & 0 & 0 & $\left.\widehat{t}_{4}(5)\right|_{x_{3}=0}=36 x_{1} x_{2}+\mathbf{1 6} x_{1}-\mathbf{2 2} x_{2}+\mathbf{0}$ \\
\hline
\end{tabular}

For $k=3$, there are six states and the calculation has been presented explicitly in Table 9. Here, we just explain the case of $s_{3}=0$.

For $s_{3}=0, s_{3}+4 \in s_{4}, x_{3}^{*}=0,1$, so Case (2b) in Procedure 4.1 is applied. If $x_{3}^{*}=0$, then $\widehat{t_{3}}\left(s_{3}\right)=\left.\widehat{t_{4}}(0)\right|_{x_{3}=0}$. Since there are multiple $\widehat{t_{4}}(0)$, we should perform $S E T_{-} 0$ according to Case 2 in Procedure 4.2 . For $x_{4}^{*}=0$ and $s_{4}=0$, we find out $\widehat{t_{4}}(0)$ with the minimum constant term and set $x_{3}=0$. For $x_{4}^{*}=1$ and $s_{4}=0$, there is only one $\widehat{t_{4}}(0)$ and we set its $x_{3}$ to 0 . If $x_{3}^{*}=1$, we perform $S E T_{-} 1$ with the same procedure.

Finally, we can obtain the optimal value and solution by the binary combination of $x_{1}$ and $x_{2}$. Refer to Table 10 . Here, $s_{3}=3 x_{1}+2 x_{2}$.

Table 10. Feasible values of $\widehat{t_{3}}\left(s_{3}\right)$

\begin{tabular}{|l|l|l|}
\hline$\left(x_{1}^{*}, x_{2}^{*}\right)=(0,0)$ & $s_{3}=0$ & $f_{00}=-20,-48,36$ \\
\hline$\left(x_{1}^{*}, x_{2}^{*}\right)=(0,1)$ & $s_{3}=2$ & $f_{01}=-22,-24,-20$ \\
\hline$\left(x_{1}^{*}, x_{2}^{*}\right)=(1,0)$ & $s_{3}=3$ & $f_{10}=16,-32$ \\
\hline$\left(x_{1}^{*}, x_{2}^{*}\right)=(1,1)$ & $s_{3}=5$ & $f_{11}=30$ \\
\hline
\end{tabular}

From Table 10, we find out $f(x)$ is minimum when $x_{1}=0$ and $x_{2}=0$. The optimal value is -48 . Then by the tracking method, we can obtain $x_{3}^{*}, x_{4}^{*}, x_{5}^{*}$ and $x_{6}^{*}$.

$$
\begin{aligned}
& s_{3}^{*}=a_{1} x_{1}^{*}+a_{2} x_{2}^{*}=3 \times 0+2 \times 0=0, \\
& x_{3}^{*}=x_{3}^{*}\left(s_{3}^{*}\right)=x_{3}^{*}(0)=0 . \\
& s_{4}^{*}=s_{3}^{*}+a_{3} x_{3}^{*}=0+4 \times 0=0, \\
& x_{4}^{*}=x_{4}^{*}\left(s_{4}^{*}\right)=x_{4}^{*}(0)=1 . \\
& s_{5}^{*}=s_{4}^{*}+a_{4} x_{4}^{*}=0+3 \times 1=3, \\
& x_{5}^{*}=x_{5}^{*}\left(s_{5}^{*}\right)=x_{5}^{*}(3)=0 . \\
& s_{6}^{*}=s_{5}^{*}+a_{5} x_{5}^{*}=3+4 \times 0=3, \\
& x_{6}^{*}=x_{6}^{*}\left(s_{6}^{*}\right)=x_{6}^{*}(3)=0 .
\end{aligned}
$$

Thus, $x^{*}=(0,0,0,1,0,0)$. 


\section{Computational Results}

In this section, we present the numerical results of the algorithms proposed to demonstrate their effectiveness and efficiency.

These algorithms were coded by $\mathrm{C}++$ and run on an Intel Dual Core PC (2.2GHz, 1.0G RAM). All the instances are generated randomly. For the dimension $n$ of $Q$ from $n=10$ to $n=300$, twelve tests have been performed and each test is the average computational time $(s)$ of 100 iterations. When calculating the average values of the ten tests, we removed the maximum and minimum data to ensure the accuracy of the calculation. Numerical results of computational time in milliseconds ( $m s$ ) for solving $U B Q P 7, U B Q P 5$ and $L C B Q P 5$ are summarized in Table 11. From this table, the considerably excellent performance in computational time of these algorithms is obvious.

Table 11. Computation time of $B Q P 7, U B Q P 5$, and $L C B Q P 5$ in milliseconds

\begin{tabular}{|c|c|c|c|c|c|c|c|c|c|c|c|c|}
\hline$n$ & $\mathbf{1 0}$ & $\mathbf{2 0}$ & $\mathbf{3 0}$ & $\mathbf{4 0}$ & $\mathbf{5 0}$ & $\mathbf{6 0}$ & $\mathbf{7 0}$ & $\mathbf{8 0}$ & $\mathbf{9 0}$ & $\mathbf{1 0 0}$ & $\mathbf{2 0 0}$ & $\mathbf{3 0 0}$ \\
\hline$U B Q P 7$ & 2.79 & 2.89 & 3.30 & 4.19 & 5.39 & 8.20 & 8.28 & 11.77 & 12.90 & 15.41 & 47.30 & 103.59 \\
\hline$U B Q P 5$ & 0.11 & 0.16 & 0.23 & 0.37 & 0.51 & 0.63 & 0.83 & 1.49 & 3.12 & 4.01 & 15.12 & 23.40 \\
\hline$L C B Q P 5$ & 3.01 & 5.28 & 6.91 & 8.76 & 10.89 & 14.13 & 19.48 & 24.19 & 30.61 & 35.41 & 132.60 & 296.70 \\
\hline
\end{tabular}

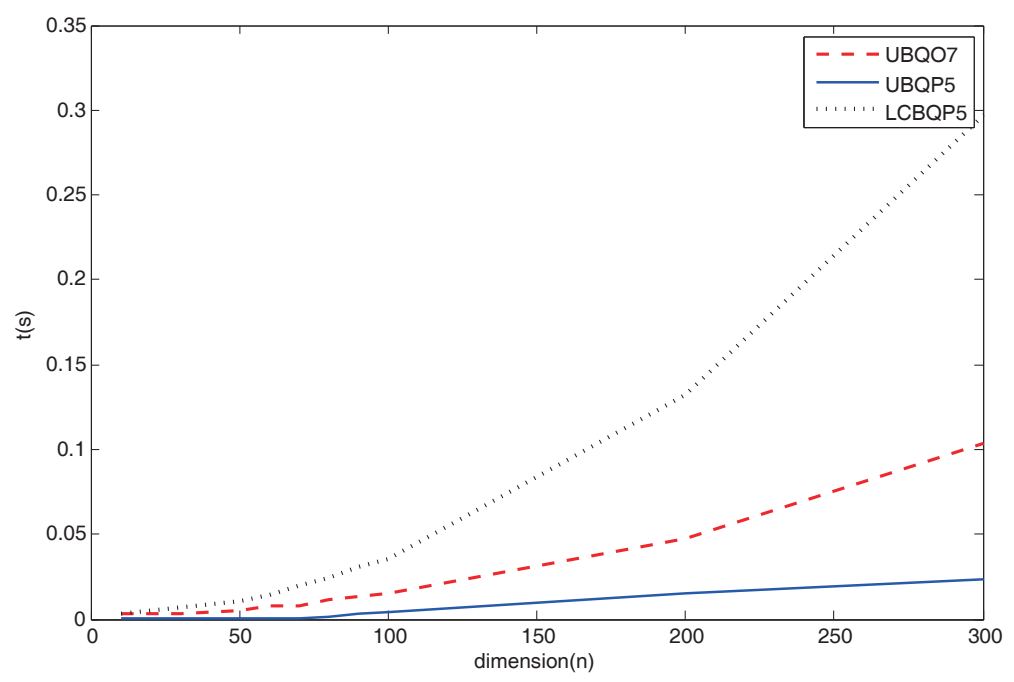

Figure 2. Average computational time for different dimensional $U B Q P 7, U B Q P 5$, and $L C B Q P 5$

We also demonstrate that our algorithm is effective and efficient with the polynomial time solvable feature. Fig.2 shows the average computational time for different dimensional $U B Q P 7, U B Q P 5$, and $L C B Q P 5$. The curves in the figure are polynomial, which obviously reveals the effectiveness and high efficiency of our algorithm.

In addition, in order to verify the upper bound of state number and cost-to-go function number, we apply our algorithm to a random $L C B Q P 5$ problem with 100 variables and obtain Fig.3. The left subfigure presents the number of states of each stage for this problem and the right one presents the number of cost-to-go functions generated at each stage.

It has been analyzed in Section 5 that, for each state $s_{k}$, there are only one cost-to-go function for Stage $k=n$, no more than two for $k=n-1$ and at most four for other stages. We can easily find out that Fig.3 reveals this important feature. That is to say, the space complexity of the Algorithm 4.1 will not increase exponentially with the dimension of the problem. 

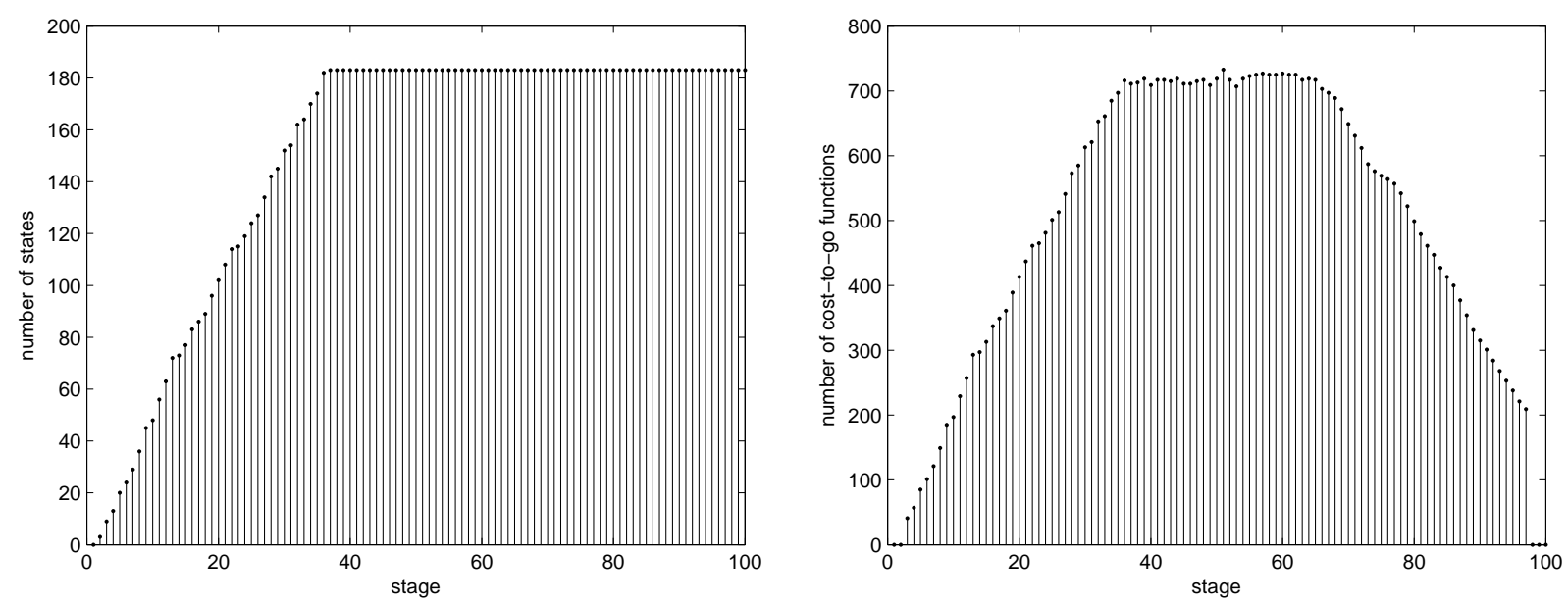

Figure 3. Number of states and cost-to-go functions at each stage for an $L C B Q P 5$ example with $\mathrm{n}=100$

\section{Conclusion}

In this paper, some novel polynomial solvable algorithms to a class of binary quadratic programming are proposed. The algorithm to $U B Q P 7$ is designed by analyzing the property of matrix $Q$. The algorithm to $U B Q P 5$ is designed based on the famous basic algorithm. And the algorithm to $L C B Q P 5$ is proposed by combining the basic algorithm and the dynamic programming method. The analysis and experimental results reveal that these novel algorithms are polynomial solvable with excellent effectiveness and high efficiency. Designing polynomially solvable algorithms to these special subclasses of problem $B Q P$ is very important. It not only provides useful information for designing efficient algorithms for other special cases of $B Q P$, but also can provide hints and facilitate the derivation of efficient relaxations for the general form of $B Q P$.

\section{Acknowledgment}

This work was supported by the Specialized Research Fund for the Doctoral Program of Higher Education (SRFDP) under Grant 20113108120010.

\section{References}

[1] M. R. Garey and D. S. Johnson, “Computers and Intractability: A Guide to the Theory of NP-Completeness," WH Freeman E Co. New York, 1979.

[2] R. D. Mcbride and J. S. Yormark, "An implicit enumeration algorithm for quadratic integer programming," Manage. Sci., vol. 26, pp. 282-296, 1980.

[3] A. T. Phillips and J. B. Rosen, "A quadratic assignment formulation of the molecular conformation problem," J. Global Optim., vol. 4, pp. 229-241, 1994.

[4] P. Chardaire and A. Sutter, "A decomposition method for quadratic zero-one programming," Manage. Sci., vol. 41, pp. 704-712, 1995

[5] D. Li and X. L. Sun, "Nonlinear Integer Programming," Springer, New York, 2006.

[6] F. Barahona, M. Jünger, and G. Reinelt, "Experiments in quadratic 0-1 programming," Math. Program., vol. 44, pp. 127-137, 1989.

[7] A. Billionnet and S. Elloumi, "Using a mixed integer quadratic programming solver for the unconstrained quadratic 0-1 problem," Math. Program., vol. 109, pp. 55-68, 2007.

[8] C. Helmberg and F. Rendl, "Solving quadratic (0,1)-problems by semidefinite programs and cutting planes," Math. Program., vol. 82, pp. 291-315, 1998.

[9] P. M. Pardalos and G. P. Rodgers, "Computational aspects of a branch-and-bound algorithm for quadratic zero-one programming," Computing., vol. 45, pp. 131-144, 1990.

[10] F. Rendl, G. Rinaldi, and A. Wiegele, "Solving max-cut to optimality by intersecting semidefinite and polyhedral relaxations," Lecture Notes Comput. Sci., vol. 4513, pp. 295-309, 2007.

[11] P. L. Hammer and S. Rudeanu, "Boolean Methods in Operations Research and Related Areas," Springer-Verlag, Berlin, Heidelberg, New York, 1968. 
[12] Y. Crama, P. Hansen, and B. Jaumard, “The basic algorithm for pseudo-Boolean programming revisited,” Discrete Appl., vol.29, pp.171-185, Math. 1990

[13] P. Hansen, B. Jaumard, and V. Mathon, “Constrained nonlinear 0-1 programming," ORSA J. Computing., vol. 5, pp. 97-119, 1993.

[14] S. S. Gu, "A polynomialtime solvable algorithm to linearly constrained binary quadratic programmingproblems with Q being a tri-diagonal matrix," Advances in Information Sciences and Service Sciences 2011., vol. 3, no. 6, pp. 65-72, 2011. 
Shenshen Gu was born in Shanghai, China. He received his B.E degree in Computer Science from Shanghai University of Engineering Science in 2002, his M.E. degree in Computer Science from Shanghai University in 2005, and his Ph.D. degree in Automation and Computer-Aided Engineering from the Chinese University of Hong Kong in 2009. He then joined the School of Mechatronics Engineering and Automation at Shanghai University, where he is currently an associate professor. Dr. Shenshen Gu's research interests include optimization, optimal control and neural networks.

Rui CUI was born in Xiangyang, Hubei, China. He received his B.E degree in Automation from Xidian University, Xi' an, in 2011. He is currently an M.Phil. student at the School of Mechatronic Engineering and Automation, Shanghai University under the supervision of Dr. Shenshen Gu. His research interests include optimization, optimal control and neural networks.

Jiao Peng was born in Loudi, Hunan, China. She received her B.E degree in Nanjing University of Posts and Telecommunications, Nanjing, in 2013. She is currently an M.Phil. student at the School Mechatronic Engineering and Automation, Shanghai University under the supervision of Dr. Shenshen Gu. Her research interests include integer programming and computational intelligence. 


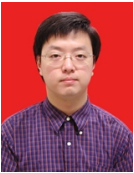




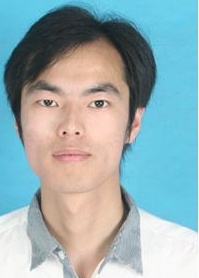




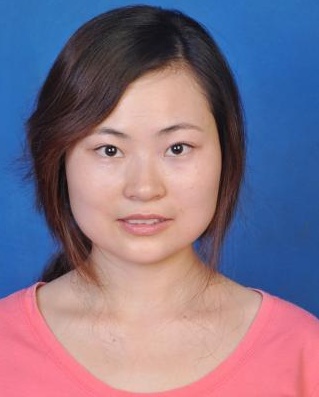

